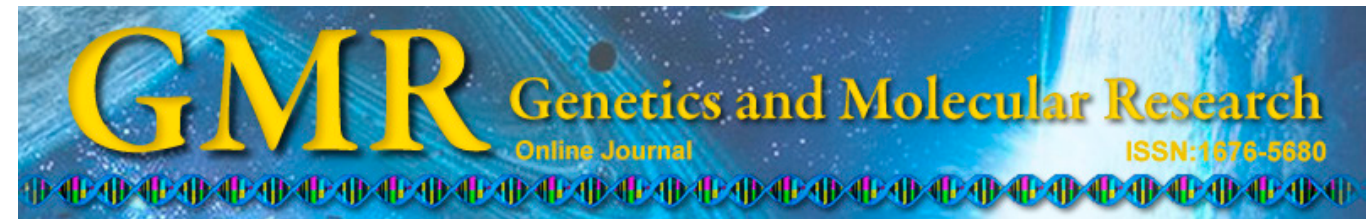

\title{
Using volume-time curves with real-time three-dimensional echocardiography to analyze right ventricular function in patients with pneumoconiosis
}

\author{
D.M. Wei ${ }^{1}$, Y. Ding ${ }^{2}$ and W. He ${ }^{1}$ \\ ${ }^{1}$ Department of Ultrasound, Beijing Tian Tan Hospital, \\ Capital Medical University, Beijing, China \\ ${ }^{2}$ Department of Radiology, Beijing Chao-Yang Hospital, \\ Capital Medical University, Beijing, China \\ Corresponding author: W. He \\ E-mail: hewen201319@163.com
}

Genet. Mol. Res. 13 (4): 9665-9674 (2014)

Received March 5, 2014

Accepted July 27, 2014

Published November 14, 2014

DOI http://dx.doi.org/10.4238/2014.November.14.12

\begin{abstract}
We evaluated right ventricular function in patients with pneumoconiosis using real-time three-dimensional echocardiography (RT3DE). A total of 80 consecutive patients were prospectively recruited, 44 of whom were diagnosed with pneumoconiosis, and the remaining 36 age- and gender-matched healthy volunteers served as the control group. All patients underwent both $2 \mathrm{D}$ and 3DE. The tricuspid regurgitation pressure (TRPG), right ventricular anterior wall thickness and range of motion, right ventricular posterior wall thickness and range of motion, right ventricular end-diastolic volume, right ventricular endsystolic volume, and right ventricular ejection fraction (RVEF) were measured. The RVEF of healthy volunteers ranged from 50 to $78 \%$, while the RVEF of pneumoconiosis patients ranged from 29 to $73 \%$. TRPG influenced RVEF by $77.3 \%(\mathrm{P}=0.006)$ and showed a negative correlation $(\mathrm{r}=-0.643, \mathrm{P}<0.01)$. Volume-time curves $(\mathrm{VTC})$ of patients
\end{abstract}


with pneumoconiosis showed more troughs (low stroke volumes) than the VTCs of normal subjects. Evaluation of right ventricular function in patients with pneumoconiosis using RT3DE can provide additional clinical information.

Key words: Right ventricular function; Volume-time curves; Real-time three-dimensional echocardiography; Pneumoconiosis

\section{INTRODUCTION}

Scientific advancements in the study of cardiac structure and function have led to increased attention on evaluation of right ventricular function (Di Salvo et al., 1995; Rigolin et al., 1995). Assessment of the degree of volume and pressure load caused by various diseases on right ventricular function (Heusch et al., 2006; Kjaergaard et al., 2006) is extremely important when making treatment choices and predicting prognosis. In two-dimensional echocardiography (2DE), right ventricular function is assumed from the left ventricle based on geometry, and then calculated from a formula for the left ventricle, so this method is prone to large errors. The main reason behind these errors is the fact that the right ventricle is an irregular, crescent-shaped structure, featuring many trabeculations, which form an irregular endocardial border. Furthermore, the inflow and outflow tracts do not exist in the same plane and are quite different in shape between the right and left ventricle. Considering these factors, processes ignoring the inflow and outflow tracts are not rigorous enough in their methodologies.

Previous studies used magnetic resonance imaging (MRI) and X-ray computed tomography (CT) on healthy individuals and patients with heart disease to evaluate ventricular volume, stroke volume, and ejection fraction (EF). MRI measurements of stroke volume and end-diastolic ventricular volume tend to underestimate the values and lack consistency (Rajappan et al., 2002), while EF can be overestimated by CT (Utsunomiya et al., 2006). Threedimensional echocardiography (3DE) (Ahmad, 2001; Utsunomiya et al., 2006), which has undergone rapid development in recent years, is based on the actual morphology of the heart chambers and involves acquiring multi-planar images and digitally reconstructing them using $3 \mathrm{D}$ technology. Without a geometric model of the left ventricle, real-time 3DE (RT3DE) can display the spatial structure of the heart chamber and measure data to form volume-time curves. RT3DE can overcome many limitations of 2DE: It not only enables observation of the morphology of the heart chambers but also assesses right ventricular stroke output and right ventricular volume parameters (Jiang et al., 1994; Muller et al., 2002; Nesser et al., 2006). The $3 \mathrm{D}$ volume is set to a fan angle of $60^{\circ} \times 60^{\circ}$ in patients with heart enlargement. The probe position must be changed to acquire the full range of the ventricular endometrial into the fan angle to avoid the loss of some portion of the volume (Endo et al., 2006).

Pneumoconiosis mainly changes the pathology of emphysema and pulmonary fibrosis associated with the final stages of chronic pulmonary heart diseases. Moreover, right ventricular function is affected by the gradual increase in pulmonary artery pressure. In this study, changes in right ventricular function were observed by RT3DE in patients with pneumoconiosis. 


\section{MATERIAL AND METHODS}

\section{General information}

The study included 36 healthy volunteers and 44 patients diagnosed with pneumoconiosis by an occupational disease diagnosis appraisal committee. Their ages ranged from 23 to 83 years, with a mean age of $57 \pm 16$ years. The healthy volunteers showed normal findings on physical examination, electrocardiography (ECG), and routine echocardiography or showed only physiological valve regurgitation. Radiographs or CT images for pneumoconiosis staging and a history of dust exposure were recorded for patients with pneumoconiosis. All 44 patients signed a consent form before undergoing radiography or CT imaging. The exclusion criteria included atrial fibrillation and pulmonary embolism. The use of clinical data was approved by the Hospital Ethics Committee.

\section{Equipment}

A Philips IE33 echocardiography instrument, which is a first-generation RT3D imaging transducer ( 2.5 or $3.5 \mathrm{MHz}$ ), was used. It consists of 256 non-simultaneously firing elements in a matrix that can be activated simultaneously to generate a pyramidal volume in $60^{\circ}$ x $60^{\circ}$ during a single heartbeat.

\section{Research methods}

\section{Image acquisition}

Subjects were placed in a left lateral position and monitored by synchronous ECG, after which the images were acquired. The imaging was visualized clearly in an apical 4-chamber view and a parasternal 4-chamber view; the right ventricle was placed at the center of the sampling area, and the gain and contrast were adjusted to clearly show the right ventricle and its intima. After full-volume imaging was initiated and the $3 \mathrm{D}$ image capture button pressed, pyramid-like full-volume 3D images were produced in a wide angle of $60^{\circ} \times 60^{\circ}$. After this acquisition, the subjects were asked to hold their breath at the end of expiration to decrease unavoidable artifacts, and the images were stored in the instrument for analysis.

Using color Doppler and M-mode echocardiography chart records, the tricuspid regurgitation pressure (TRPG), right ventricular anterior wall thickness and range of motion $\left(\mathrm{TH}_{1}, \mathrm{M}_{1}\right)$, and right ventricular posterior wall thickness and range of motion $\left(\mathrm{TH}_{2}, \mathrm{M}_{2}\right)$ were measured.

\section{Image analysis}

The QLAB 3DQ Adv measurement software was used to analyze right ventricular function. Based on the end-diastolic and end-systolic values from the ECG, the endocardial border of the tricuspid annulus and right ventricular apex were marked, and the software 
automatically drew an outline of the right ventricular intima. The next step was to select a sequence to analyze the stored images by frames/s. Ultimately, we obtained data such as the right ventricular end-diastolic volume, right ventricular end-systolic volume, right ventricular ejection fraction (RVEF), and volume-time curve (VTC). In the VTCs (left ventricular VTC), the peak indicates the ventricular end-diastolic volume, and the trough indicates the end-systolic ventricular volume. It also contains information about the heart ventricular volume over 4 cardiac cycles.

\section{Statistical analysis}

The SPSS version 11.0 software was used for the analysis, and the data are reported as means \pm standard deviation for normally distributed values. RVEF and tricuspid regurgitation pressure (TR) were compared using a 2 -sample $t$-test, multi-factor analysis of variance, and multiple correlation analysis. The cut-off value for significance was set at 0.05 .

\section{RESULTS}

\section{Staging of patients with pneumoconiosis by radiography}

Among the 44 pneumoconiosis patients, 18 cases were classified as phase I $(22.5 \%), 19$ phase II $(23.8 \%)$, and the remaining 7 cases $(8.8 \%)$, phase III. The range of dust exposure history was less than 1 year to 39 years. Twenty-eight cases showed a TRPG $\leq 25 \mathrm{mmHg}$, while the other 16 cases showed a TRPG $\geq 26 \mathrm{mmHg}$. The $\mathrm{TH}_{1}$ and $\mathrm{TH}_{2}$ values showed significant differences $(\mathrm{P}<0.01)$ between the pneumoconiosis patients and healthy subjects, but not their $\mathrm{M}_{1}$ or $\mathrm{M}_{2}$ values $(\mathrm{P}>0.05)$ (Table 1$)$.

In this study, the RVEF values of healthy volunteers ranged from 50 to $78 \%$, and those of pneumoconiosis patients between 29 and $73 \%$ (Table 2).

After multi-factor analysis of variance, we found that TRPG had an influence on RVEF amounting to $77.3 \%$, with a significant difference $(P=0.006)$, but no significant difference was observed in RVEF values from a history of dust exposure or imaging stage $(\mathrm{P}>0.05)$. TR and RVEF showed a negative correlation in a multivariate analysis $[\mathrm{r}=-0.643(<0.01)]$.

Ejection fraction (EF) was found to be decreased and TRPG, significantly increased in 2 patients, but their history of dust exposure and pneumoconiosis stage were significantly different (see Figures 1-6).

From the extent of the TRPG, we attempted to divide the pneumoconiosis patients into 2 subgroups: pressure-compensated (subgroup $\mathrm{A}_{1}$, TRPG $<26 \mathrm{mmHg}$ ) and pressuredecompensated (subgroup $\mathrm{A}_{2}, \mathrm{TRPG} \geq 26 \mathrm{mmHg}$ ) subgroups. In subgroup $\mathrm{A}_{1}$, there were 28 cases, whose RVEF ranged from 50 to $71 \%$. In the other 16 cases in subgroup $A_{2}$, the RVEF varied between 29 and $73 \%$.

According to the RVEF, we categorized the pneumoconiosis patients into function compensated (subgroups $\mathrm{B}_{1}, \mathrm{RVEF} \geq 50 \%$ ) and decompensated (subgroup $\mathrm{B}_{2} \mathrm{RVEF}<50 \%$ ) groups. There were 35 cases with TRPG below $47 \mathrm{mmHg}$ in subgroup $\mathrm{B}_{1}$ and 9 cases with TRPG between 45 and $97 \mathrm{mmHg}$ in subgroup $\mathrm{B}_{2}$. 
Table 1. Right ventricular wall thickness and mean and range of motion (in $\mathrm{mm}$ ).

\begin{tabular}{lccccc}
\hline Group & Cases & $\mathrm{TH}_{1}$ & $\mathrm{M}_{1}$ & $\mathrm{TH}_{2}$ & $\mathrm{M}_{2}$ \\
\hline Healthy & 36 & $3.27 \pm 0.65$ & $6.23 \pm 0.99$ & $3.81 \pm 0.66$ & $8.02 \pm 1.73$ \\
Pneumoconiosis & 44 & $4.07 \pm 0.91$ & $6.58 \pm 2.54$ & $5.46 \pm 1.43$ & $7.39 \pm 1.79$ \\
$\mathrm{P}$ & & 0.000 & 0.433 & 0.000 & 0.114 \\
\hline
\end{tabular}

$\mathrm{TH}_{1}=$ right ventricular anterior wall thickness; $\mathrm{M}_{1}=$ right ventricular anterior wall range of motion; $\mathrm{TH}_{2}=$ right ventricular posterior wall thickness; $\mathrm{M}_{2}=$ right ventricular posterior wall range of motion.

Table 2. Right ventricular volume and mean EF and TR values on three-dimensional ultrasonography.

\begin{tabular}{lcccc}
\hline Group & TRPG $(\mathrm{mmHg})$ & RVEDV $(\mathrm{mL})$ & RVESV $(\mathrm{mL})$ & RVEF \\
\hline Healthy & $2.33 \pm 6.75$ & $25.68 \pm 11.42$ & $9.25 \pm 5.22$ & $64 \% \pm 8 \%$ \\
Pneumoconiosis & $25.27 \pm 24.97$ & $40.10 \pm 22.77$ & $18.65 \pm 14.57$ & $55 \% \pm 11 \%$ \\
P & 0.000 & 0.001 & 0.000 & 0.000 \\
\hline
\end{tabular}

TRPG = tricuspid regurgitation pressure; RVEDV = right ventricular end-diastolic volume; RVESV $=$ right ventricular end-systolic volume; RVEF = right ventricular ejection fraction.

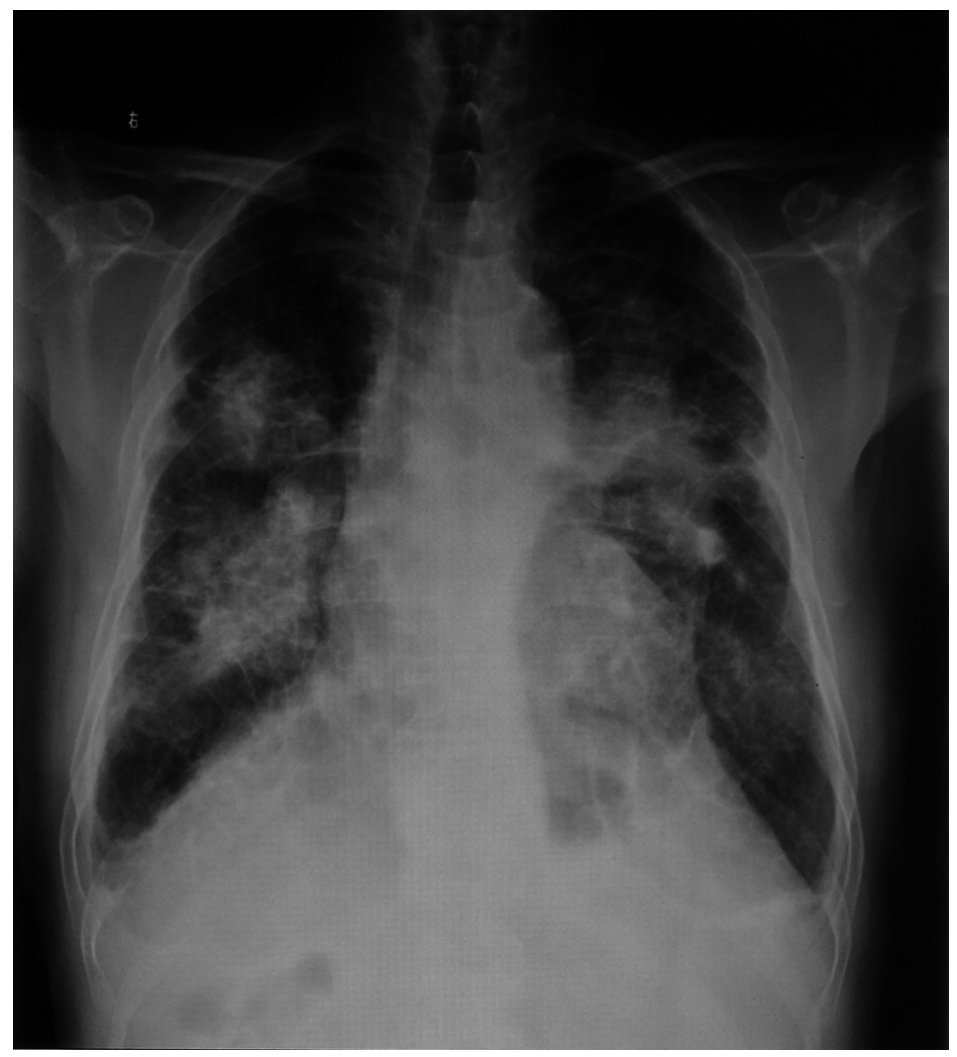

Figure 1. Patient: A 70-year-old man with a history of 20 years of dust exposure. The radiograph shows 2 markings of weight gain and small nodules in both lungs in oval and irregular dense shadow; part of the boundary is clear, multiple shadowy streaks are visible, and cystoid translucent film is present. The diagnosis was phase III pneumoconiosis. 


\section{Global Function Report}

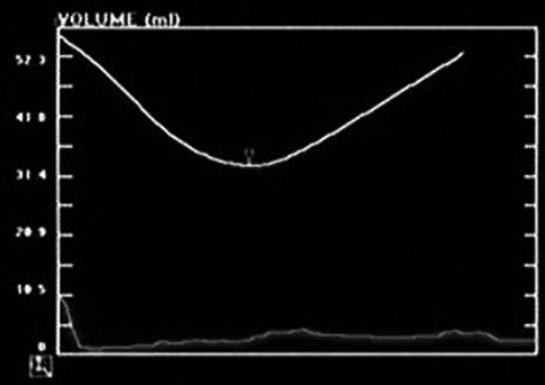

HR $\quad 100 \mathrm{bpm}$

Systolic Function

EDV ost $57.5 \mathrm{mt}$

ESV est $33.6 \mathrm{mI}$

SV ost $24.0 \mathrm{ml}$

If ost $41.7 \times$

Figure 2. Real-time three-dimensional echocardiographic volume-time curve image of the same patient in Figure 1 shows an ejection fraction of $41.7 \%$.

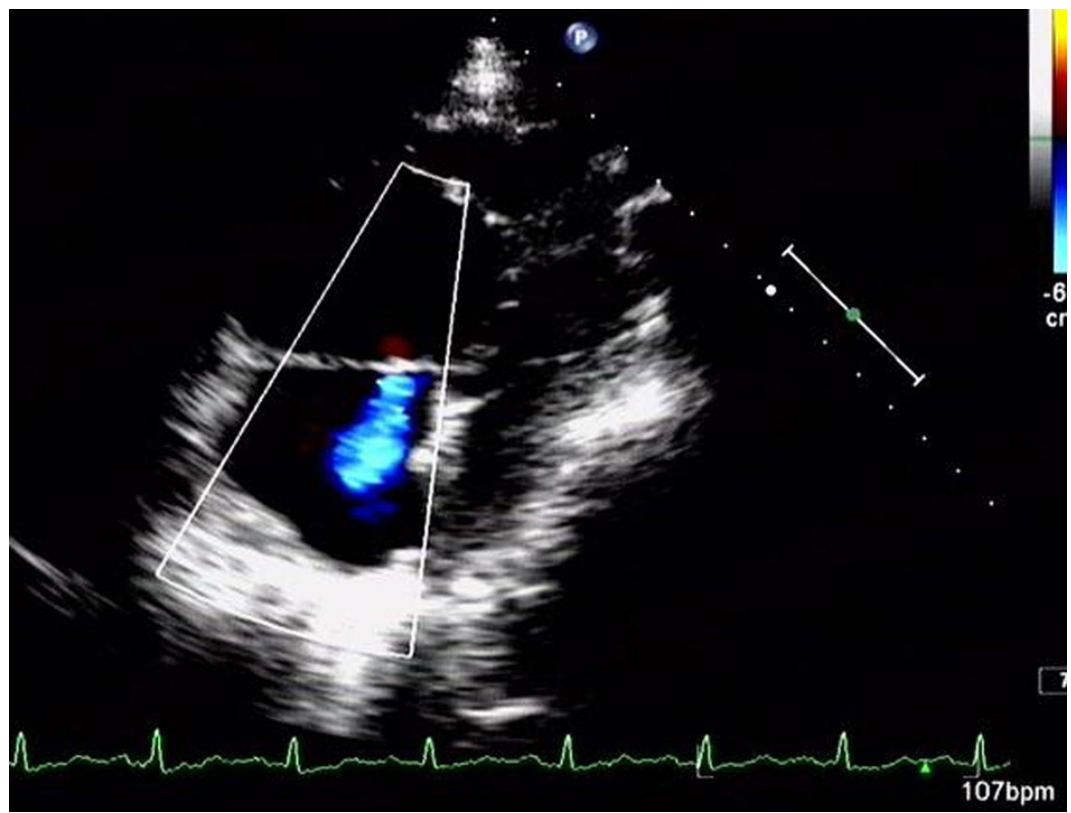

Figure 3. Two-dimensional color Doppler sonogram of the same patient in Figure 1 shows right heart increaseand mild regurgitation of the tricuspid valve (the tricuspid regurgitation pressure was $72 \mathrm{mmHg}$ ). 


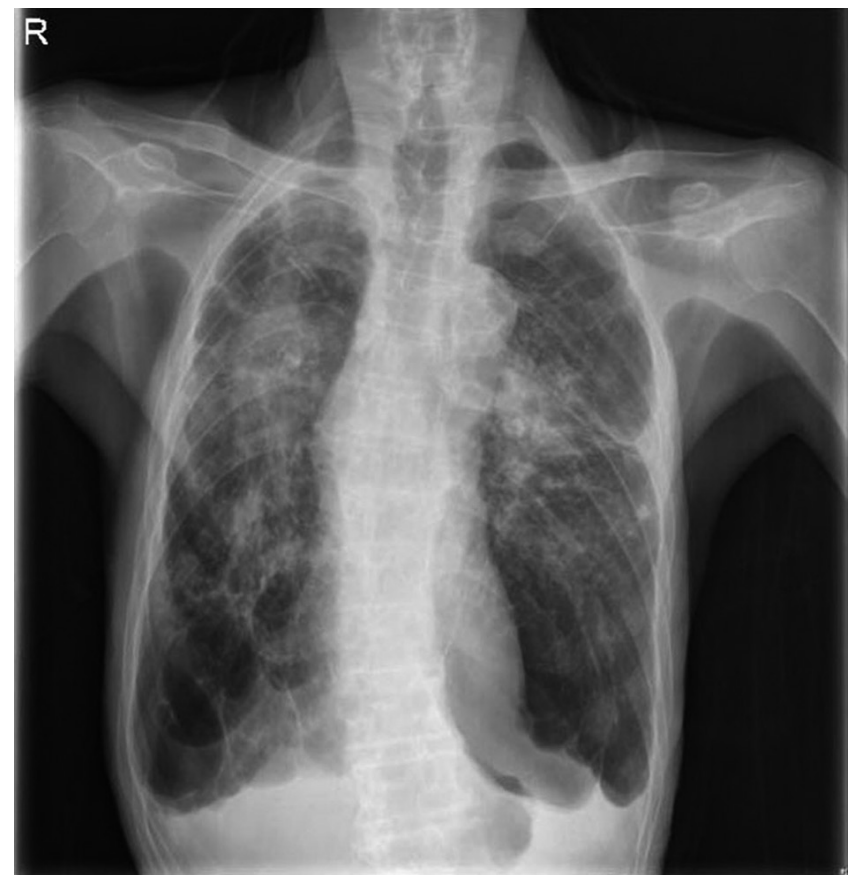

Figure 4. Patient: An 82-year-old man with a history of 33 years of dust exposure. Radiographic image pairs show heavy markings, disorders, dual lung fields with multiple fiber cable of the shadow, partial fusion of the reticular nodules, scattered calcification, double opacities in the lung fields, visible lumps on the sides of the apex pairs, step down by degrees increased, and the left lung door's lymph node calcification. The diagnosis was phase III pneumoconiosis.

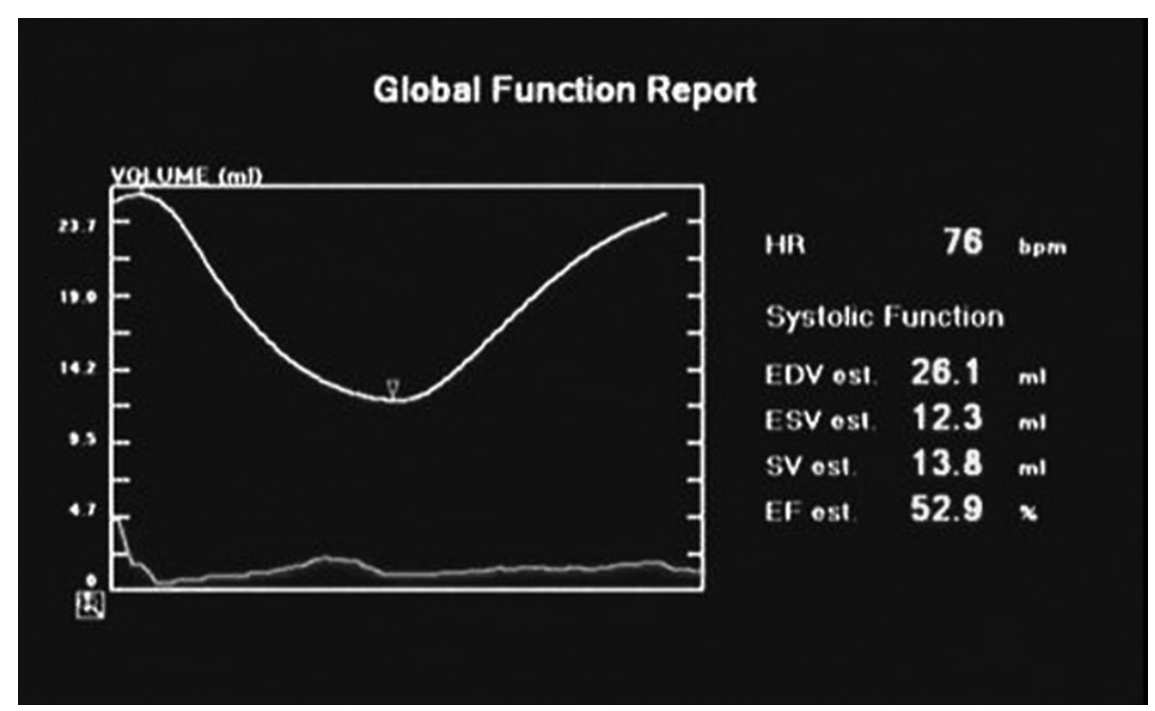

Figure 5. Real-time three-dimensional echocardiographic volume-time curve image of the same patient in Figure 4 shows an ejection fraction of $52.9 \%$. 


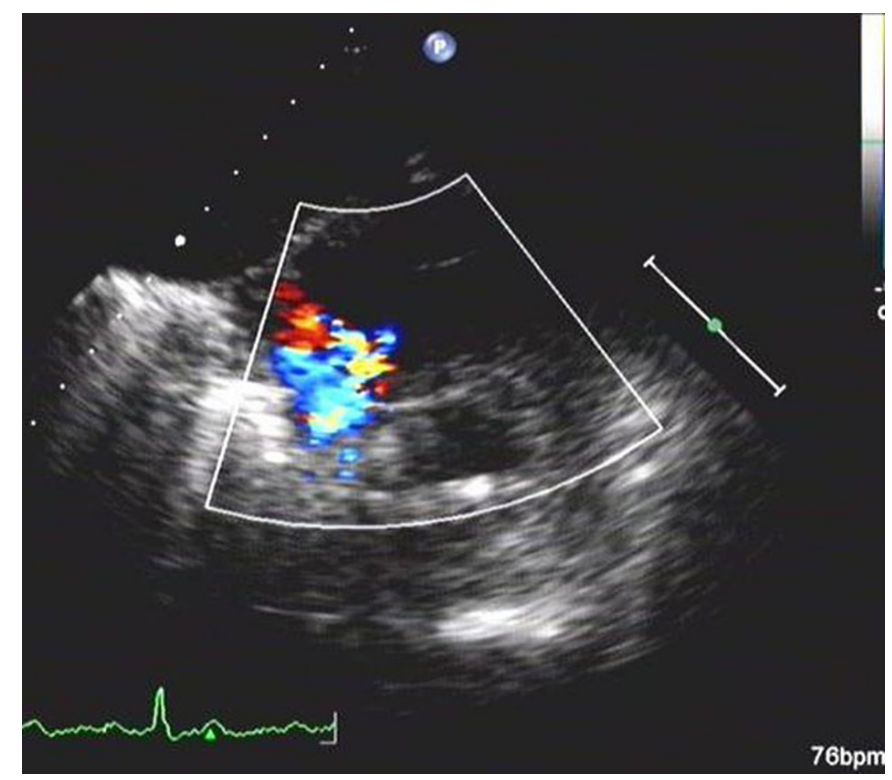

Figure 6. Two-dimensional color Doppler sonogram of the same patient in Figure 4 shows mild regurgitation of the tricuspid valve (the tricuspid regurgitation pressure was $40 \mathrm{mmHg}$ ).

\section{DISCUSSION}

Pneumoconiosis is an occupational lung disease characterized by chronic inflammation and fibrosis caused by the inhalation of mineral dust or metal. According to the diagnostic criteria formulated by the Code of Occupational Disease Prevention of the People's Republic of China, the diagnosis of pneumoconiosis involves an occupational history of dust exposure, respiratory symptoms and signs, and staging by radiography; however, right heart hemodynamic changes secondary to pulmonary fibrosis are not mentioned. This study focused on right ventricular function changes in different stages of pneumoconiosis. It was found that a history of dust exposure and radiography staging are not enough to reflect changes in right ventricular function. Interestingly, TRPG had an influence on RVEF of 77.3\% $(\mathrm{P}=0.006)$; RVEF tends to decrease with an increase in TRPG, showing a negative correlation. Right ventricular pressure load affected the volume load. An increase in right ventricular pressure load leads to right ventricular compensatory hypertrophy. An enhancement in right ventricular volume load results in significant morphological changes to the right ventricle. Significant differences were found in $\mathrm{TH}_{1}, \mathrm{TH}_{2}, \mathrm{TRPG}, \mathrm{EF}$, and right ventricular volume between pneumoconiosis patients and healthy subjects. These changes of ultrasonography are similar to pulmonary heart disease of other causes. The RVEF is the main indicator of right ventricular function. Owing to the wide use of RT3D ultrasonography in recent years, more attention has been paid to the right ventricular configuration and its cardiac output measurements (Jiang et al., 1994; Endo et al., 2006; Nesser et al., 2006). Researchers (Yan et al., 1995) discovered that the RVEF of patients with pulmonary heart disease was less than that of healthy subjects through $3 \mathrm{D}$ ultrasonography. In this study, the lowest RVEF observed was $29 \%$ among the pneumoconiosis patients. After a series of changes in the right heart from advancing pulmonary artery pressure, right 
ventricular systolic and diastolic functions deteriorate (Hao et al., 1999). The present study used TRPG to determine the changes in pulmonary artery pressure, while Arcasoy et al. (2003) showed that the correlation coefficient between ultrasonography and right heart catheterization to measure pulmonary artery systolic pressure was 0.69 , and reported the sensitivity of ultrasonography as $85 \%$, with a specificity of $55 \%$. In normal left ventricular VTCs (Zeidan et al., 2003), the deeper the trough the greater the stroke volume and the greater the difference between end-diastolic and end-systolic volume, which is reflected by the heart effectively pumping out more blood. In general, the right ventricle acts only as a channel, but from the view of the right ventricle VTCs, the right ventricle also plays a part in the pumping of blood. Previous studies suggested that right ventricular systolic function was reduced in the compensatory stage of pulmonary heart disease (Liu et al., 2008). In our study, the RVEF of subgroup $A_{1}$ was still in the normal range; while in subgroup $\mathrm{A}_{2}$, the RVEF ranged from normal to significantly reduced. The TRPG was also mildly elevated in subgroup $B_{1}$, and the TRPG of subgroup $B_{2}$ showed mild to severe elevation. This implies that the evaluation of the function of the right heart without TRPG and RVEF is not comprehensive. When TRPG exists in the normal range (TRPG $<26 \mathrm{mmHg}$ ), the RVEF is normal. When the RVEF is reduced $(<50 \%)$, the TRPG varied slightly or was severely elevated. However, there were variations between pressure and function compensations when the TRPG was elevated $(\geq 26 \mathrm{mmHg})$ and when RVEF was increased $(\geq 50 \%)$. In this study, the planes of the apical and parasternal 4-chamber heart were used in the methodology, including the right ventricular inflow tract (tricuspid valve annulus) while excluding the right ventricular outflow tract below the pulmonary valve. Researchers believe that the diameter of the right ventricular inflow tract changes significantly during the systolic and diastolic stages, which have a major influence on the right ventricular stroke volume. In contrast, the right ventricular outflow tract (from the ventricle to the pulmonary valve) is a uniform tubular funnel and has minor variations so that the VTC is not affected. The only changes are that the right ventricular end-diastolic volume and end-systolic volume are slightly smaller. Overall, the hemodynamics of the right heart in pneumoconiosis is variable. RT3DE is an option in determining critical conditions.

\section{Conflicts of interest}

The authors declare that they have no conflict of interest.

\section{REFERENCES}

Ahmad M (2001). Real-time three-dimensional echocardiography in assessment of heart disease. Echocardiography 18: 73-77.

Arcasoy SM, Christie JD, Ferrari VA, Sutton MS, et al. (2003). Echocardiographic assessment of pulmonary hypertension in patients with advanced lung disease. Am. J. Respir. Crit. Care Med. 167: 735-740.

Di Salvo TG, Mathier M, Semigran MJ and Dec GW (1995). Preserved right ventricular ejection fraction predicts exercise capacity and survival in advanced heart failure. J. Am. Coll. Cardiol. 25: 1143-1153.

Endo Y, Maddukuri PV, Vieira ML, Pandian NG, et al. (2006). Quantification of right ventricular volumes and function by real time three-dimensional echocardiographic longitudinal axial plane method: validation in the clinical setting. Echocardiography 23: 853-859.

Hao LW, Zhou TH and Wang X (1999). Study to the right heart function of chronic pulmonary heart disease. Chin. J. Intern. Med. 38: 264.

Heusch A, Lawrenz W, Olivier M and Schmidt KG (2006). Transesophageal 3-dimensional versus cross-sectional echocardiographic assessment of the volume of the right ventricle in children with atrial septal defects. Cardiol. Young 16: 135-140. 
Jiang L, Siu SC, Handschumacher MD, Luis Guererro J, et al. (1994). Three-dimensional echocardiography. In vivo validation for right ventricular volume and function. Circulation 89: 2342-2350.

Kjaergaard J, Snyder EM, Hassager C, Oh JK, et al. (2006). Impact of preload and afterload on global and regional right ventricular function and pressure: a quantitative echocardiography study. J. Am. Soc. Echocardiogr. 19: 515-521.

Liu Y, Chen M and Zheng JH (2008). Real-time three-dimensional echocardiography in assessment of right ventricular systolic function by volume curves of chronic pulmonary heart disease. Chin. J. Ultrasonogr. 16: 91.

Muller S, Bartel T, Pachinger O and Erbel R (2002). 3-D echocardiography: new developments and future prospects. Herz 27: $227-236$.

Nesser HJ, Tkalec W, Patel AR, Masani ND, et al. (2006). Quantitation of right ventricular volumes and ejection fraction by three-dimensional echocardiography in patients: comparison with magnetic resonance imaging and radionuclide ventriculography. Echocardiography 23: 666-680.

Rajappan K, Livieratos L, Camici PG and Pennell DJ (2002). Measurement of ventricular volumes and function: a comparison of gated PET and cardiovascular magnetic resonance. J. Nucl. Med. 43: 806-810.

Rigolin VH, Robiolio PA, Wilson JS, Harrison JK, et al. (1995). The forgotten chamber: the importance of the right ventricle. Cathet. Cardiovasc. Diagn. 35: 18-28.

Utsunomiya D, Tomiguchi S, Awai K, Shiraishi S, et al. (2006). Mutidetector-row CT and quantitative gated SPECT for the assessment of left ventricular function in small hearts: the cardiac physical phantom study using a combined SPECT/CT system. Eur. Radiol. 16: 1818-1825.

Yan ZN, Huang DJ and Wu JH (1995). A study to assess the right ventricular function with three dimensional echocardiography. Chin. J. Ultrasonogr. 4: 36.

Zeidan Z, Erbel R, Barkhausen J, Hunold P, et al. (2003). Analysis of global systolic and diastolic left ventricular performance using volume-time curves by real-time three-dimensional echocardiography. J. Am. Soc. Echocardiogr. 16: $29-37$ 